\title{
Editorial \\ Systems Engineering: Availability and Reliability
}

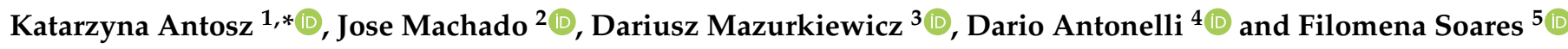 \\ 1 Faculty of Mechanical Engineering and Aeronautics, Rzeszow University of Technology, \\ Aleja Powstańców Warszawy 8, 35-959 Rzeszow, Poland \\ 2 MEtRICs Research Center, Department of Mechanical Engineering, School of Engineering, University of \\ Minho, 4800-058 Guimarães, Portugal; jmachado@dem.uminho.pt \\ 3 Mechanical Engineering Faculty, Lublin University of Technology, Nadbystrzycka 36, 20-618 Lublin, Poland; \\ d.mazurkiewicz@pollub.pl \\ 4 Department of Management and Production Engineering, Polytechnic University of Turin, \\ Corso Duca degli Abruzzi 24, 10138 Torino, Italy; dario.antonelli@polito.it \\ 5 Centro Algoritmi, Campus of Azurém, University of Minho, 4800-058 Guimaraes, Portugal; \\ fsoares@dei.uminho.pt \\ * Correspondence: katarzyna.antosz@prz.edu.pl
}

Citation: Antosz, K.; Machado, J.; Mazurkiewicz, D.; Antonelli, D.;

Soares, F. Systems Engineering: Availability and Reliability. Appl. Sci. 2022, 12, 2504. https://doi.org/ 10.3390/app12052504

Received: 9 February 2022

Accepted: 22 February 2022

Published: 28 February 2022

Publisher's Note: MDPI stays neutral with regard to jurisdictional claims in published maps and institutional affiliations.

Copyright: (C) 2022 by the authors. Licensee MDPI, Basel, Switzerland. This article is an open access article distributed under the terms and conditions of the Creative Commons Attribution (CC BY) license (https:// creativecommons.org/licenses/by/ $4.0 /)$.

\section{Introduction}

Current trends in Industry 4.0 are largely related to issues of reliability and availability. As a result of these trends and engineering systems' complexity, research and development needs now refer to new solutions in the integration of intelligent machines or systems, with emphasis on changes in production processes aimed at increasing production efficiency or equipment reliability. The emergence of innovative technologies and new business models based on innovation, cooperation networks, and the enhancement of endogenous resources is assumed to be a strong contribution to the development of competitive economies all around the world. Innovation and engineering, focused on sustainability, reliability, and availability of resources, have a key role in this context. The scope of this Special Issue is closely associated with that of the ICIE'2020 conference. This conference and the journal's Special Issue aims to present the current innovations and engineering achievements of top world scientists and industrial practitioners in the thematic areas related to:

- Reliability and risk assessment;

- Innovations in maintenance strategies;

- Production process scheduling, management and maintenance;

- Systems analysis, simulation, design and modeling.

The rapid development of industry, especially observed in recent decades, has significantly complicated the functioning of production systems and thus intensified the maintenance process. Nowadays, the reliability of hardware resources and employee safety, as well as low environmental risk, is required while implementing production processes with maximum efficiency, effectiveness and flexibility. At the same time, the awareness of the role of maintenance services in preventing breakdowns and early detection of initial problems with machines and systems is growing, which, in turn, increases the life of production systems, affecting the achievement of a high level of productivity.

Detecting and diagnosing faults in the early stages of damage is necessary to prevent the incorrect operation of the machine park and its breakdowns during operation. Hence, the growing role of availability and reliability research. Regular monitoring of the actual technical condition of the equipment and the operational efficiency of technological systems allows ensuring the maximum interval between repairs, thus extending the time of effectively using the existing hardware resources. It also minimizes the number and cost of unplanned downtime caused by machine failures, increasing the availability of both individual machines and entire production lines. It also allows making the right decisionrepair or replacement-using cost-benefit analysis methods using appropriate optimization 
tasks, for example, when determining the maintenance strategy of the machine as a whole or separately for each category of its structural elements. The implementation of such tasks, also based on the methodology of optimal maintenance, requires appropriate knowledge about the course of operation of a given machine or its element in the past, currently and in a certain forecast of future use and its impact on potential failures and overall service life. Measurement systems monitoring the operation of machines and their parameters as well as diagnostic systems are thus extremely helpful. They all record significant amounts of data that require appropriate processing into knowledge and inference. An extremely dynamic digital transformation is taking place here.

\section{Reliability and Risk Assessment}

With the rapid development of high integrations in large complex systems, such as aircraft, satellite and railway systems, due to the increasingly complex coupling relationship between components within the system, local disturbances or faults may cause global effects on the system by fault propagation. Therefore, there are new challenges in safety analysis and risk assessment for complex systems. Some of them with their proposed solutions are described below.

As presented in [1], to this day, research articles highlighting analytical methods on small data sets are practically limited. From the literature review presented [1], it is revealed that most of the studies conducted thus far using scarce failure data are less. The methods used for reliability analyses for small number data are mostly the Bayesian approach, FMECA and Monte Carlo method. Using these methods, the small failure data of any machine or system can be grouped and easily used for further reliability analysis. Maintenance philosophies involve performing maintenance after given time intervals, typically after a fixed running hour for an engine. In spite of the scheduled maintenance, failure of the engine is inevitable, thereby decreasing the availability of dumpers and reducing the production cost. Reliability analysis of engine subsystems is essential for formulating the maintenance strategies that will reduce the downtime of the engine and enhance its availability. The main obstacle was the deficiency of adequate data for the appropriate statistical analyses. A data set containing a small sample size of failure data limits the possibility of precise decision-making. The study [1] gives specific guidelines for using $\mathrm{CBH}$ and meta-analysis testing, which emphasizes the failure data to predict reliability and MTBF. The researchers perform the reliability assessment using the grouped TBF data, during which suitable maintenance strategies could be formed. It provides a roadmap of reliability analysis for any machinery having fewer failure data.

For the development of the technical solution-a reliable robotic system to facilitate precision and safety-the ISO 14971 standard for risk management in healthcare devices was followed in [2]. This paper presents the risk management strategy for the development of the technical solution. Moreover, by evaluating the associated risks of the procedure, the medical and technical requirements for the proposed technical solution are defined. It also presents an analytical hierarchy process that is introduced subsequently in a four-phase quality function deployment, which, in turn, leads to the HeRo conceptual design, showing the advantages of the design with respect to risk reduction. The residual risks introduced by the robotic system were also evaluated, showing a reduction in hazard occurrence and severity, which validates the HeRo concept as a possible technical solution.

An approach based on the hidden Markov model is proposed [3] for risk performance reasoning. The unobservable state process in the approach aims to model the underlying risk performance, while the observation process was formed from the time series of risk factors. Within the framework, the log-likelihood probability was used as the measure of similarity between historical and current data of risk reasoning factors. Based on scalar quantization regulation and risk performance quantization regulation, the risk performance reasoning approach with different step sizes was conducted on the operational case, the performance of which was evaluated in terms of effectiveness and accuracy. The results obtained show significant improvement in the reasoning capacity and satisfactory 
performance for numerical risk reasoning and categorical performance reasoning. The proposed model is able to provide a reference for risk performance monitoring and threat pre-warning during the bauxite shipping process. The risk performance analyzed in [3] is essential in relation to the bauxite shipping process, whose associated risk remains to be officially recognized. The output of this paper can support quantitative risk decision-making, as opposed to previously used empirical decision-making, thereby laying a foundation for risk pre-warnings and process safety.

Aiming at analyzing and evaluating the inherent risks of the complex system with coupling correlation characteristics objectively, Jiang et al. [4] propose a novel risk assessment and analysis method for correlations in the complex system based on multi-dimensional theory. Firstly, the formal description and coupling degree analysis method of the hierarchical structure of complex systems is established. Moreover, considering the three safety risk factors of fault propagation probability, potential severity, and fault propagation time, a multi-dimensional safety risk theory is proposed in order to evaluate the risk of each element within the system affecting the overall system. Furthermore, critical safety elements are identified based on Pareto rules, As Low as Reasonably Practicable and safety risk entropy to support the preventive measures. Finally, an application of an avionics system is provided to demonstrate the effectiveness of the proposed method. Compared with the current methods and technologies, the method proposed [4] mainly reflects the advantages of two aspects. On the one hand, the hierarchical model is modeled in a matrix manner, and the association relationship of each element in the complex system is quickly and accurately analyzed, which reduces the skill requirements of analysts. On the other hand, it provides a feasible and multi-faceted analysis method for the risk assessment of systems in view of fault propagation, which is the core judgment criterion for identifying critical risk factors and is of great significance for ensuring system safety.

\section{Innovations in Maintenance Strategies}

The key issue in the use of technical facilities is the problem of making optimal decisions. Along with the development of computer aiding techniques and the progressive digitization of production, numerous tools have appeared to facilitate the work of technical services, allowing for the collection and processing of data, information and knowledge about objects and operational processes, thus enabling and facilitating effective decisions regarding the technical systems in use. This applies to both the technical, organizational and economic areas.

Optimally made decisions are one of the key conditions for the proper functioning of maintenance services, which means the necessity to determine the value of selected features. Correct exploitation of technical infrastructure facilities requires linking decisionmaking processes with the performance of maintenance or repair work. There are many mathematical models that constitute the basis for the quantitative assessment of the method and scope of the operation of technical objects. However, there are still several research challenges requiring innovations in maintenance strategies.

For example, as mentioned in [5], the supply chain of petroleum products faces major challenges, i.e., demand growth and the complexity of fluid transportation. The petroleum supply chain contains multiple stations extending from oil wells in exploration and production areas to the final destination. Each station has its own difficulties and challenges in the contribution towards the success of the safe and continuous supply. Undoubtedly, meeting a regional demand for petroleum products requires an uninterrupted, safe operation. In addition, the unloading of petroleum products is a complex and potentially dangerous operation since the unloading system contains complex interdependency components. Any failures in one of its components lead to a cut in the petroleum supply chain. Therefore, it is important to assess and evaluate the reliability of the unloading system in order to improve its availability. In this context, Mohammed et al. [5] present the operation philosophy of the truck unloading system, failure modes of the components within the system, and a bottom-up approach to analyze the reliability of the system. In addition, it provides reliabil- 
ity data, such as failure rates and the mean time between failures of the system components. Furthermore, the reliability of the whole system was calculated and is presented for different time periods. The critical components, which are major contributors towards the system's reliability, were identified. To enhance the system's reliability, a reliability-based preventive maintenance strategy for the critical components was implemented. In addition, the preventive maintenance scheduling was identified based on the reliability plots of the unloading system. The best schedule for preventive maintenance of the system was determined based on the reliability function to be every 45 days for maintaining the system reliability above 0.9 .

In order to maximize inventory benefits or minimize costs, the reliability and cost of inventory control models need to be identified and analyzed. These importance measures are one important approach to recognize and evaluate system weaknesses. However, the importance measures have fewer applications in inventory systems' reliability. Considering the cost, Chen et al. [6] discuss the reliability change of performance parameters with the importance measures in inventory systems. The calculation methods of differential importance and Birnbaum importance are studied in the inventory control model with shortages. By comparing the importance values of various parameters in the model, the optimization analysis of the inventory model can be used to identify the key parameters so as to effectively reduce the total inventory cost. The importance order and the identification of key parameters are helpful to increase the operational efficiency of the inventory control and provide effective methods for improving inventory management. Lastly, a case study with a shortage and limited inventory capacity is used to demonstrate the proposed model. One of the most important contributions of this paper [6] is that based on the research of inventory systems, it was found that there was almost no literature on the reliability of an inventory system. Combining the concept of reliability with the inventory system, an inventory system reliability model was proposed. It could enrich the research in the field of inventory system reliability.

The growing competitiveness of the market, coupled with the increase in automation driven by the advent of Industry 4.0, highlights the importance of maintenance within organizations. At the same time, the amount of data capable of being extracted from industrial systems has increased exponentially due to the proliferation of sensors, transmission devices and data storage via the Internet of Things. These data, when processed and analyzed, can provide valuable information and knowledge about the equipment, allowing a move towards predictive maintenance being considered one of the most innovative maintenance strategies. Maintenance is fundamental to a company's competitiveness since actions taken at this level have a direct impact on aspects such as cost and quality of products. Hence, equipment failures need to be identified and resolved. Artificial Intelligence tools, in particular Machine Learning, exhibit enormous potential in the analysis of large amounts of data, now readily available, thus aiming to improve the availability of systems, reduce maintenance costs and increase operational performance and support in decision making. This is why Cardoso and Ferreira [7] apply Machine Learning to a set of data made available online and the specifics of this implementation are analyzed, as well as the definition of methodologies, in order to provide information and tools to the maintenance area. Although the results obtained compare well with those presented so far in the literature, the biggest disadvantage in using the presented methodology lies in the definition of the features. If the selection of features is not the most correct, the results obtained can lead to wrong predictions. For future work, the application of feature learning concepts will be considered instead of feature engineering, which appears to be promising to improve the results obtained [7]. This section may be divided into subheadings. It should provide a concise and precise description of the experimental results, their interpretation, as well as the experimental conclusions that can be drawn. 


\section{Production Systems Scheduling, Management and Maintenance}

Advancements in technology, such as information and communication technologies, have changed the traditional manufacturing systems practices. This is especially true for different manufacturing systems due to their ability to cater to their needs such as Big Data, interoperability, timely delivery, etc.

Production and maintenance tasks apply for access to the same resources. Maintenancerelated machine downtime reduces productivity, but the costs incurred due to unplanned machine failures often outweigh the costs associated with predictive maintenance. Costs incurred due to unplanned machine failure include corrective maintenance, reworks, delays in deliveries, breaks in the work of employees and machines. Therefore, scheduling production and maintenance tasks should be considered jointly.

The problem of generating a predictive schedule with given constraints is considered by Paprocka et al. [8], with an objective to develop a scheduling method that reflects the operation of the production system and the nature of disturbances. The original value research results presented is the development of the method of a basic schedule generation with the application of Ant Colony Optimization. A predictive schedule is built by planning the technical inspection of the machine at the time of the predicted failure-free time. The numerical simulations are performed for job/flow shop systems. In the future, the presented method for generating predictive schedules will be compared with the genetic algorithm, as well as immune and clonal selection algorithms. The presented algorithm may, however, contribute to the development of a method that reflects the operation of the production system and the nature of disturbances and improves the system operation.

In production systems maintenance, fault propagation behavior analysis is the basis of fault diagnosis and health maintenance. As presented by $\mathrm{Mu}$ et al. [9], traditional fault propagation studies are mostly based on a priori knowledge of a causality model combined with rule-based reasoning, disregarding the limitations of experience and the dynamic characteristics of the system that cause deviations in the identification of critical fault sources. Thus, the authors [9] propose a dynamic analysis method for fault propagation behavior of machining centers that combines fault propagation mechanisms with model structure characteristics. They use the design structure matrix to establish the fault propagation hierarchy structure model. Considering the correlation of fault time, the fault probability function of a component is obtained, and the fault influence degree of nodes are calculated. By introducing the Copula and Coupling degree functions, the fault influence degree of the edges between the same level and different levels are calculated, respectively. As a result, a fault propagation intensity model was constructed by integrating the edge betweenness and then used as an index to analyze real-time fault propagation behavior. Finally, a certain type of machining center is taken as an example for a specific application. This study can provide a reference for the fault maintenance and reliability growth of a machining center. According to the fault propagation intensity of the components, the critical fault propagation paths and nodes of a machining center can be identified, provide a reference for the fault maintenance and encourage reliability growth of machining centers. This paper [9] demonstrates the effectiveness and practicability of the proposed method through the application of the specific case.

Ramakurthi et al. [10] point out that rising energy prices, increasing maintenance costs and strict environmental regimes have augmented the already existing pressure on the contemporary manufacturing environment. Although the decentralization of the supply chain has led to rapid advancements in manufacturing systems, finding an efficient supplier simultaneously from the pool of available ones as per customer requirements and enhancing the process planning and scheduling functions are the predominant approaches that still need to be addressed. Therefore, they have decided [10] to address this important issue by considering a set of gear manufacturing industries located across India as a case study. An integrated classifier-assisted evolutionary multi-objective evolutionary approach is proposed for solving the objectives of makespan, energy consumption and increased service utilization rate, interoperability and reliability. To execute the approach initially, 
text-mining-based supervised machine-learning models were adopted for the classification of suppliers into task-specific suppliers. Following this, with the identified suppliers as input, the problem was formulated as a multi-objective Mixed-Integer Linear Programming model, and finally, a Hybrid Multi-Objective Moth Flame Optimization algorithm was proposed to optimize process planning and scheduling functions. Numerical experiments have been carried out with the formulated problem as well.

\section{Systems Analysis, Simulation, Design and Modeling}

The activities performed by the maintenance department are usually a combination of technical, administrative and management activities carried out during the life cycle of a given technical object. The effective implementation of these activities requires appropriate data management-data collection, proper analysis and the use of appropriately effective models to support decision making. We use the data for many different purposes, e.g., to determine the moment of generating service orders, monitor the quality of performed activities, optimize and plan activities or develop plans for the delivery dates of materials and spare parts, i.e., in the field of broadly understood logistics for the operation of technical facilities and systems.

This is why proper data management and knowledge management are extremely important issues for the implementation and effective maintenance strategies when it is possible not only to determine the current wear of devices or their structural elements but also to predict whether and when a failure or catastrophic wear will occur. It is also possible to effectively detect the cause of a failure or identify performance or product quality problems. To achieve this, adequate analysis, design and modeling methods are required.

This is why Aliev and Antonelli [11] have focused on developing a framework using I4.0 enabling technologies to improve reliability and safety in human-robot collaboration applications. The proposed framework allows a robot's condition to be continuously monitored during human-robot collaboration. The monitoring deploys IoT connectivity, a data acquisition system, physical cyber-systems and ML tools to perform analytics. The paper is divided as follows: the relevant equipment parameters are first identified, a description of the data acquisition framework is then given, an application to an assembly case study in which all the necessary data are collected is presented, and finally, the analysis results of the considered case study are presented and discussed. The case study was performed on benchmark tasks for collaborative assembly processes. An automatic machine learning tool was used to perform online monitoring and predict outages of the industrial robots during a human-robot collaboration process. Such an online monitoring system allows more reliable human-robot collaboration applications to be created, unplanned downtime during task execution to be eliminated, and the trust of humans during interaction with a robot and the lifetime of the robot to be maximized. The proposed framework demonstrates data management techniques on an industrial robot that is considered as a physical=cyber system.

Achievements of accurate robotic arm-based bike frame quality checks with the use of a 3D mathematical model are discussed by Lin et al. [12]. Unlike the traditional way to find coefficients of a space sphere, the proposed model requires only three check point coordinates to achieve the sphere axis coordinate and its radius. In the practical work, the contact sensor combined with the robotic arm is used to realize the compliance items measurement in shaft length, internal diameter, verticality, parallelism, etc. The proposed model is validated based on both mathematic verification and an actual bike frame check. The stylus probe used in the proposed model presents a simple and accurate performance. However, successful measurement depends on the activity range of the robotic arm that certain features of bike frames should be reached by the stylus probe. In future work, the optical sensors may provide an alternative solution, although more complex signal processing algorithms should be addressed.

The quality and reliability of consumables, including gear oils, resulting in the failurefree operation of the transmission components in heavy trucks are discussed by Gil et al. [13]. 
As oil viscosity is essential for all lubricated tribopairs for wear and friction reduction in all vehicles with a gearbox, it may be influenced by the contamination that wear products can impart on the oil. Oil contamination can also affect lubrication efficiency in the boundary friction conditions in gearboxes where slips occur (including bevel and hypoid gearboxes). Therefore, the present research [13] focused on this issue. An obvious hypothesis was adopted, where it was theorized that exploiting the contaminants that are present in gear oil may affect how the lubricating properties of gear oils deteriorate. Laboratory tests were performed on contaminants that are commonly found in gear oil. The study was designed to identify a number of different solid particles that are present in oil. The quantitative contamination of the gear oils that contained solid particles and the curves representing the friction coefficients of fresh oils with a history of exploitation were compared. Exploitation was shown to have a significant impact on the contamination of gear oils. It was revealed that the contamination and the mileage had no effect on the tested oils. The research [13] showed that the presence of contaminants is not catastrophic and that in order to fully examine the oils and to determine the critical moment, the oils with a much greater operational mileage should be tested in order to establish the relationship between the number of particles and their tribological properties.

Various factors are considered in system design, such as efficiency, costs, safety, and environmental effect. Availability is also one of the important issues in system design. The availability indicates how much a system approaches ideal operation without production loss caused by equipment failures or undesired external events. Availability estimation is frequently performed in the oil and gas, chemical and power plant industries to find the optimum design option, to predict the production level and to evaluate maintenance and operating policies. Precise availability estimation is important because it directly influences the owner's decision. This is why Seo et al. [14] have investigated the availability gap between the early and late design stages by estimating it with the design stages to find a practical manner of availability estimation in the early design stage. The sensitivity analysis was conducted to analyze the key factors in the results. The most crucial factor was the redundant equipment. Although this study [14] did not consider the whole system, this gives an important guide to progress the next step for the accurate availability estimation in the early design stage.

Steel tapes with certain special markings generated on their surface are often used for metrological and technological purposes, for example, to measure displacement. The smoothness of the movement of a moving precision tape and the stability of the tape area where symbols are generated are very important factors that affect the quality parameters of a generated tape. One of the most important characteristics of the raster generation device is the smoothness of the stretching of the tape, which is important for the overall operation of the system. The smoothness of the stretching of the tape affects errors in the position of the raster element being generated and the control of activation of the laser beam. Determining not only the amplitude but also the frequency of the belt stretching oscillations is important. For this purpose, [15] analyzes the tape movement system consisting of electromechanical tape pulling and its constant stretching mechanisms as well as a tape deflection mechanism, which operates in sliding friction. This system was mounted on a massive granite base placed on a foundation using passive vibration insulation supports. A research and data processing method together with the results of experimental research of a mock-up system were developed and presented to examine the raster generation method and the generation device. This method may be used to produce a precision metrological scale on stainless steel tape. The generation process takes place in the dynamic mode because both the steel tape and the laser raster generation head are constantly moving during the process. The main aim of the research [15] was to develop a system for measuring the displacement of the tape in the raster generation device, to examine the model of that system and to evaluate the possible impact of external and internal factors on raster generation in the dynamic mode. 
The use of numerical methods for simulation, design or modeling, such as finite element analysis (FEA), has proven to be an advantageous tool to predict the mechanical behavior of many materials. Up to now, the application of FEA related to elastomers and other cork composites has been utilized to access static, dynamic loading and impact behavior. One of the first requirements for the application of isolation pads is to evaluate their capacity to support static loadings. Regarding elastomers, one of the crucial steps during FEA is the definition of material properties. Typically for large strains, elastomer's properties are defined through the application of non-linear models. However, if a linear stress-strain relationship at small strains is observed, Hooke's Law can be adopted for that strain range. As expressed by Lopes et al. [16], like other types of elastomers, different geometries of the same cork-rubber material present different mechanical behavior when subject to compression between bonded plates. To validate the application of Hooke's Law on cork-rubber materials, under compression at small strains, a set of experimental and numerical analyses has been conducted [16]. Using finite element analysis, a methodology is described to relate frictionless and frictional compression between a cork-rubber sample and loading plates. Based on that, the performance of square cross-section blocks with other dimensions can be evaluated. The results obtained by this approach [16] showed a good agreement with experimental compression tests and with outputs from other models available in the literature relating Young and apparent compression moduli. However, future research should address the effect of higher shape factors, other cross-section shapes (rectangular and other polygons), and the friction coefficient between sample and loading surfaces. Moreover, applying this knowledge and relating it to the dynamic compression behavior of isolation pads could be a topic of interest.

\section{Conclusions}

The availability and reliability of engineering systems is an important issue for modern companies, especially from the Industry 4.0 challenges and requirements. In addition, in recent years, the concept of sustainable development has also been gaining importance, as sustainable, intelligent production should be taken into account. This means developing products manufactured through production processes that have a minimal negative impact on the environment, save energy and natural resources and are safe for workers and the community and economically viable. This means that the goal of sustainable production is to achieve a balance between the environmental, social and economic dimensions. Creating a sustainable production environment also requires the elimination of breakdowns and energy waste and, as a concept, is strongly associated with sustainable maintenance. From a practical point of view, this requires changes in the activities performed in the maintenance area. Overall, this means the ability to monitor, control and process data combined to create intelligent, learning, self-diagnosing and self-adapting technology machines. This kind of technological intelligence in maintenance can reduce the need for operators to act, improving safety and reducing unnecessary costs; however, they can be still considered an important research challenge in systems engineering.

Funding: This research received no external funding.

Institutional Review Board Statement: Not applicable.

Informed Consent Statement: Not applicable.

Data Availability Statement: Not applicable.

Conflicts of Interest: The authors declare no conflict of interest. 


\section{References}

1. Dinkar, B.; Mukhopadhyay, A.; Chattopadhyaya, S.; Sharma, S.; Alam, F.; Machado, J. Statistical Reliability Assessment for Small Sample of Failure Data of Dumper Diesel Engines Based on Power Law Process and Maximum Likelihood Estimation. Appl. Sci. 2021, 11, 5387. [CrossRef]

2. Pisla, D.; Calin, V.; Birlescu, I.; Hajjar, N.; Gherman, B.; Radu, C.; Plitea, N. Risk Management for the Reliability of Robotic Assisted Treatment of Non-resectable Liver Tumors. Appl. Sci. 2020, 10, 52. [CrossRef]

3. Wu, J.; Jin, Y.; Hu, S.; Fei, J.; Zhang, Y. Approach to Risk Performance Reasoning with Hidden Markov Model for Bauxite Shipping Process Safety by Handy Carriers. Appl. Sci. 2020, 10, 1269. [CrossRef]

4. Jiang, Z.; Zhao, T.; Wang, S.; Ren, F. A Novel Risk Assessment and Analysis Method for Correlation in a Complex System Based on Multi-Dimensional Theory. Appl. Sci. 2020, 10, 3007. [CrossRef]

5. Mohammed, A.; Ghaithan, A.; Al-Saleh, M.; Al-Ofi, K. Reliability-Based Preventive Maintenance Strategy of Truck Unloading Systems. Appl. Sci. 2020, 10, 6957. [CrossRef]

6. Chen, L.; Kou, M.; Wang, S. On the Use of Importance Measures in the Reliability of Inventory Systems, Considering the Cost. Appl. Sci. 2020, 10, 7942. [CrossRef]

7. Cardoso, D.; Ferreira, L. Application of Predictive Maintenance Concepts Using Artificial Intelligence Tools. Appl. Sci. 2021, 11, 18. [CrossRef]

8. Paprocka, I.; Krenczyk, D.; Burduk, A. The Method of Production Scheduling with Uncertainties Using the Ants Colony Optimisation. Appl. Sci. 2021, 11, 171. [CrossRef]

9. Mu, L.; Zhang, Y.; Liu, J.; Zhai, F.; Song, J. Dynamic Analysis Method for Fault Propagation Behaviour of Machining Centres. Appl. Sci. 2021, 11, 6525. [CrossRef]

10. Ramakurthi, V.; Manupati, V.; Machado, J.; Varela, L. A Hybrid Multi-Objective Evolutionary Algorithm-Based Semantic Foundation for Sustainable Distributed Manufacturing Systems. Appl. Sci. 2021, 11, 6314. [CrossRef]

11. Aliev, K.; Antonelli, D. Proposal of a Monitoring System for Collaborative Robots to Predict Outages and to Assess Reliability Factors Exploiting Machine Learning. Appl. Sci. 2021, 11, 1621. [CrossRef]

12. Lin, H.; Yu, B.; Wang, J.; Lai, J.; Wu, J. Achievement of Accurate Robotic Arm-based Bike Frame Quality Check Using 3D Geometry Mathematical Model. Appl. Sci. 2019, 9, 5355. [CrossRef]

13. Gil, L.; Przystupa, K.; Pieniak, D.; Kozlowski, E.; Antosz, K.; Gauda, K.; Izdebski, P. Influence of Contamination of Gear Oils in Relation to Time of Operation on Their Lubricity. Appl. Sci. 2021, 11, 11835. [CrossRef]

14. Seo, Y.; Jung, J.; Han, S.; Kang, K. Availability Estimation of Air Compression and Nitrogen Generation Systems in LNG-FPSO Depending on Design Stages. Appl. Sci. 2020, 10, 8657. [CrossRef]

15. Fursenko, A.; Kilikevicius, A.; Kilikeviciene-, K.; Borodinas, S.; Kasparaitis, A.; Matijosius, J. Investigation of Roller-Tape Contact Pair Used in Precision Mechatronic System. Appl. Sci. 2020, 10, 4041. [CrossRef]

16. Lopes, H.; Silva, S.; Machado, J. Analysis of the Effect of Shape Factor on Cork-Rubber Composites under Small Strain Compression. Appl. Sci. 2020, 10, 7177. [CrossRef] 